\title{
Влияние гидростатического давления на статическую диэлектрическую проницаемость германия
}

\author{
(C) A.М. Мусаев \\ Институт физики им. Х.И. Амирханова Дагестанского научного центра Российской академии наук, \\ 367003 Махачкала, Россия \\ E-mail: akhmed-musaev@yandex.ru
}

(Получена 28 июня 2016 г. Принята к печати 22 мая 2017 г.)

\begin{abstract}
Экспериментально исследована зависимость статической диэлектрической проницаемости монокристаллического германия от гидростатического давления до $P \approx 7.4$ ГПа. При росте давления до $P \approx 4$ ГПа, диэлектрическая проницаемость Ge уменьшается в $\sim 13$ раз до значения $\varepsilon=1.22$. При дальнейшем росте давления до $P \approx 7$ ГПа наблюдается умеренный рост $\varepsilon$ до исходного значения, а в диапазоне 7-7.4ГПа диэлектрическая проницаемость возрастает до значения более 1000 единиц. Полученные экспериментальные результаты существенно отличаются от ранее известных зависимостей.
\end{abstract}

DOI: 10.21883/FTP.2018.01.45315.8359

О фундаментальном электрофизическом параметре монокристаллического $\mathrm{Ge}$ как зависимости статической диэлектрической проницаемости от всестороннего давления $\varepsilon(P)$ до настоящего времени нет достоверной информации. Исследование зависимости $\varepsilon(P)$ при потенциале взаимодействия атомов на расстояниях, меньших чем равновесные, представляет интерес для достаточно широкого круга задач физики и техники полупроводников. В настоящее время имеется незначительное количество работ по исследованию зависимости $\varepsilon(P)$ германия, которые приведены в монографиях $[1,2]$. Эти экспериментальные результаты существенно отличаются от результатов, приведенных в работе [3], а также от результатов, полученных в последней экспериментальной работе [4], где приведена зависимость $\varepsilon(P)$ до давления 8 ГПа.

Во всех экспериментальных работах зависимость поляризуемости от давления определена косвенным методом, основанным на формуле Лоренц-Лорентца, где поляризуемость вещества $(\alpha)$ связана с показателем преломления $n$ следующей зависимостью:

$$
R=V\left(n^{2}-1\right) /\left(n^{2}+2\right)=(4 / 3) \pi N \alpha,
$$

где рефракция $R$ выражается в единицах объема $V$ ( $\mathrm{cm}^{3} /$ моль $)$, поляризуемость $\alpha=3 R / 4 \pi N=$ $=3 / 9630 \cdot 10^{-25} R$.

В этих работах зависимость статической диэлектрической проницаемости $\varepsilon(P)$ получена посредством экстраполяции частотной зависимости экспериментальных коэффициентов преломления $n(\omega)$ к нулевым частотам. В работе [4] показано, что изменение статической диэлектрической проницаемости $\varepsilon(P)$ для $\mathrm{Ge}$, связанной с изменением объема полупроводника с давлением, составляет $\Delta V / V_{0} \approx 8 \%$ и зависимость $\varepsilon(P)$ можно представить коэффициентом $r=d \ln (\varepsilon) / d \ln (V)=1.58$. $\mathrm{B}$ работе [5] получено, что значение коэффициента $\eta \equiv \partial \ln (\chi) / \partial \ln (d)$ в зависимости от межатомного расстояния $d$ составляет 2.4 .
Как известно, диэлектрическая проницаемость кристаллов, в которых связь между атомами выполняется путем обменного взаимодействия валентных электронов, значительно превышает значение $\left(n^{2}\right)$. Замена $(\varepsilon)$ на $\left(n^{2}\right)$ подразумевает, что показатель преломления является константой, измеренной в бесконечно длинных волнах. Поэтому результаты вычислений сильно зависят от точности экстраполяции показателя преломления на нулевые частоты.

В данной работе впервые получена экспериментальная зависимость статической диэлектрической проницаемости $\mathrm{Ge}$ от всестороннего давления посредством прямого экспериментального измерения. Исследование зависимости $\varepsilon(P)$ проводилось по методике, основанной на регистрации зависимости барьерной емкости $p-n$-структур от давления, на структурах со ступенчатым изменением концентрации примесей $N_{p} \gg N_{n}$ (сплавные структуры с базой из полупроводника $n$-типа) [6]. При этом зависимость статической диэлектрической проницаемости имеет вид

$$
\varepsilon=\left(1.44 \cdot 10^{8} C_{b}^{2} U_{k}\right) / \varepsilon_{0} S^{2} \cdot N_{d},
$$

где $\varepsilon_{0}=8.85416 \cdot 10^{-2}$ пф/см.

Из соотношения (2), по экспериментальным данным: $C_{b}(P)$ и известным величинам: $U_{k}(P), S(P)$, можно определить зависимость $\varepsilon(P)$. Энергия ионизации мелких примесей от давления фактически не изменяется [7], и составляет примерно $10^{-13}$ эВ/Па, тогда как изменение ширины запрещённой зоны $d E_{g} / d P=5 \cdot 10^{-11}$ эВ/Па. Расчет изменения контактного потенциала $U_{k}(P)$ с давлением производился с использованием известных коэффициентов давления $[7,8]$ для экстремумов зон проводимости $\gamma_{C i}\left(L, \Delta_{1}\right)$ и валентной зоны $\gamma_{\Delta}$ которые составляют для $\mathrm{Ge}$ :

$$
\begin{gathered}
\gamma_{C L}=83 \mathrm{мэ} / Г П а, \quad \gamma_{C \Delta}=8 \mathrm{мэВ/ГПа,} \gamma_{V}=28 \mathrm{мэВ/ГПа,} \\
\Delta E_{g(V-C L)}=55 \text { мэВ/ГПа, } \quad(P=0-2.5 \text { ГПа }), \\
\Delta E_{g(V-C \Delta)}=-20 \text { мэВ/ГПа, } \quad(P \geq 2.5 \text { ГПа }) .
\end{gathered}
$$




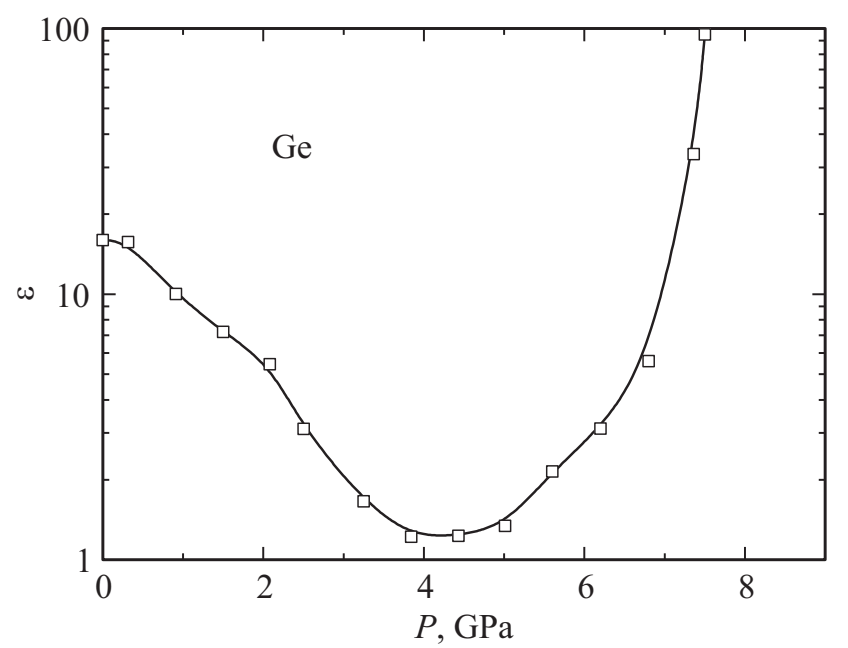

Экспериментальная зависимость статической диэлектрической проницаемости Ge от всестороннего давления.

Исследуемые $p-n$-структуры изготавливались вплавлением сплавов $\mathrm{In}-\mathrm{Pb}-\mathrm{Ga}(\mathrm{Pb}-10 \%, \mathrm{Ga}-0.7 \%$, $\mathrm{In}-89.3 \%)$ и $\mathrm{Pb}-\mathrm{Sn}-\mathrm{Sb}(\mathrm{Pb}-65 \%, \mathrm{Sn}-$ $30 \%, \mathrm{Sb}-5 \%)$ в $n$-Ge, с концентрацией примесей $N_{D}-N_{A}=4.0 \cdot 10^{14} \mathrm{~cm}^{-3}$, кристаллографически ориентированной по направлению [100].

Для генерирования давления в диапазоне 0-10ГПа применялся аппарат высокого давления типа „плоские наковальни с лункой и тороидальной поддержкой“ [9]. Барические зависимости барьерной емкости регистрировались на частоте 1 МГц при уровне измерительного сигнала 0.1 В. При расчете $\varepsilon(P)$, учитывалось изменение $C(P)$, связанное с изменением геометрических размеров структур с давлением $\Delta V(P) / V_{0}$, которое составляет $-0.25 \% /$ Па.

На рисунке представлена зависимость статической диэлектрической проницаемости для $\mathrm{Ge}$ от всестороннего давления при температуре $300 \mathrm{~K}$. Как видно из результатов эксперимента, на начальном участке наблюдается незначительный рост диэлектрической проницаемости. Дальнейшее увеличение давления приводит к существенному уменышению статической диэлектрической проницаемости, в $\sim 13$ раз до значения $\varepsilon=1.22$ при $P \approx 4$ ГПа. В диапазоне давлений 4.0-7 ГПа наблюдается умеренный рост $\varepsilon$ до исходного значения, а в диапазоне 7-7.4ГПа диэлектрическая проницаемость увеличивается до значений более 1000 единиц.

Известно, что в Ge гибридные орбитали обладают более низкой симметрией, чем составляющие их атомные орбитали, а распределение электронной плотности смещено от атомного ядра в направлении симметрии гибридной орбитали. При этом поляризуемость атомов по порядку величины совпадает с объемом атома, т.е. атомы с большим радиусом электронной орбиты легко поляризуются. Уменышение межатомных

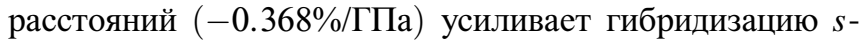

и p-орбиталей и обеспечивает большее перекрытие электронных орбит соседних атомов, при этом потенциал в центре между взаимодействующими атомами в точке ветвления не изменяется и равен нулю.

Изменение статической диэлектрической проницаемости в Ge при всестороннем давлении связано со следующими взаимно конкурирующими факторами.

1. При всестороннем давлении кулоновское отталкивание атомов и эффект наложения электронных оболочек соседних атомов приводят к увеличению кинетической энергии связывающих электронов. При этом скорость валентных электронов с уменьшением радиуса их орбит увеличивается как

$$
V_{P}=V_{0} \cdot r_{0} / r_{P}
$$

где $V_{0}-$ скорость электрона при $P=0, r_{0}-$ радиус электрона при $P=0, r_{P}-$ радиус электрона при всестороннем давлении.

Так как электронная поляризация обратно пропорциональна квадрату скорости электронов $P \approx k / V_{e}^{2}$, то с уменьшением межатомных расстояний уменьшается и коэффициент диэлектрической проницаемости.

2. Электрическое поле связующих атомов имеет точку ветвления в середине линии их связи. В точке ветвления напряженность поля равна нулю, а эквипотенциальная поверхность пересекает сама себя. Изменение электрического потенциала в зависимости от радиуса электрона при всестороннем давлении $r_{P}$ и от расстояния $h$, в направлении, перпендикулярном линии, соединяющей атомы, можно представить как [10]

$$
\varphi_{\perp} \approx-k \cdot h^{2} / r_{P}^{3},
$$

где $k-$ коэффициент пропорциональности.

Из данного соотношения следует, что потенциал связующих атомов в направлении, перпендикулярном линии их связи, с уменьшением межатомного расстояния понижается, что должно привести к увеличению влияния внешнего поляризующего электрического поля.

3. Увеличению диэлектрической проницаемости способствует и фактор повышения плотности электронов, локализованных в пространстве между атомами с уменьшением межатомных расстояний.

Из сказанного следует, что уменьшение поляризации в $\mathrm{Ge}$ при давлениях до 4 ГПа связано с преобладающим воздействием фактора увеличения кинетической скорости электронов с давлением. Последующее увеличение поляризации при $P \geq 4$ ГПа обусловлено насыщением кинетической энергии носителей и уменьшением напряженности поля в пространстве между взаимодействующими атомами, а также повышением плотности электронов, локализованных в пространстве между атомами.

Вышеприведенные объяснения наблюдаемого изменения статической диэлектрической проницаемости Ge от всестороннего давления относятся к диапазону давлений до начала их структурных фазовых переходов. По данным авторов работы [11] (см. также ссылки в 
этой работе), фазовый переход в Ge от структуры типа алмаза (I) к структуре типа $\beta$-Sn (II) происходит в диапазоне давлений 6.7-10.6 ГПа.

Исследование выполнено при финансовой поддержке РФФИ в рамках научного проекта № 15-02-05181.

\section{Список литературы}

[1] В. Пол, Д. Варшауэр. Твердые тела под высоким давлением (М., Мир, 1966) с. 524.

[2] M. Cardona, H. Paul, H.S. Brooks. Phys. Chem. Solids, 8, 204 (1959).

[3] У. Харрисон. Электронная структура и свойства твердых тел (М., Мир, 1983) т. 1, с. 383.

[4] A.R. Goñi, K. Syassen, M. Cardona. Phys. Rev., B, 41 (14), 10104 (1990).

[5] С.Ю. Давыдов, Е.И. Леонов. ФТТ, 29 (10), 2890 (1987).

[6] Л.С. Берман. Емкостные методы исследования полупроводников (Л., Наука, 1972) с. 104.

[7] А.Л. Полякова. Дебормация полупроводников и полупроводниковых приборов (М., Энергия, 1979) с. 168.

[8] П.И. Баранский, В.Г. Клочков, И.В. Потыкевич. Полупроводниковая электроника (Киев, Наук. думка, 1975) c. 704 .

[9] L.G. Khvostantsev, V.A. Sidorov. Phys. Status Solidi A, 46, 305 (1978).

[10] В.А. Говорков. Электрические и магнитные поля (М., Энергия, 1968) с. 488.

[11] М.М. Александрова, В.Д. Бланк, С.Г. Буга. ФТТ, 35 (5), 1308 (1993).

Редактор А.Н. Смирнов

\section{The influence of hydrostatic pressure on the static dielectric permittivity of germanuim}

\section{A.M. Musaev}

Institute of Physics,

Daghestan Scientific Center,

Russian Academy of Sciences,

367003 Makhachkala, Russia

Abstract The dependence of the static dielectric permittivity of monocrystalline germanium on the hydrostatic pressure up to $P \approx 7.4 \mathrm{GPa}$ is researched. When increasing the pressure up to $P \approx 4 \mathrm{GPa}$ dielectric permittivity of Ge decreases up to the value $\varepsilon=1.22$, by a factor of $\sim 13$. Further increase in pressure up to $P \approx 7 \mathrm{GPa}$ triggers a moderate rise in $\varepsilon$ to an initial value, and over the range of $7-7.4 \mathrm{GPa}$ the dielectric permittivity rises as high as 1000 units. Obtained experimental data are substantially different from previous dependences. 